\title{
Variability of Forebrain Commissures in Callosal Agenesis: A Prenatal MR Imaging Study
}

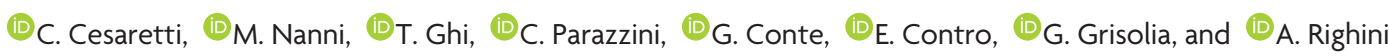

\begin{abstract}
BACKGROUND AND PURPOSE: Agenesis of the corpus callosum, even when isolated, may be characterized by anatomic variability. The aim of this study was to describe the types of other forebrain commissures in a large cohort of randomly enrolled fetuses with apparently isolated agenesis of the corpus callosum at prenatal MR imaging.
\end{abstract}

MATERIALS AND METHODS: All fetuses with apparent isolated agenesis of the corpus callosum undergoing prenatal MR imaging from 2004 to 2014, were evaluated for the presence of the anterior or a vestigial hippocampal commissure assessed in consensus by 2 pediatric neuroradiologists.

RESULTS: Overall, 62 cases of agenesis of the corpus callosum were retrieved from our data base. In 3/62 fetuses (4.8\%), no forebrain commissure was visible at prenatal MR imaging, 23/62 fetuses (37.1\%) presented with only the anterior commissure, and 20/62 fetuses (32.3\%) showed both the anterior commissure and a residual vestigial hippocampal commissure, whereas in the remaining $16 / 62$ fetuses (25.8\%), a hybrid structure merging a residual vestigial hippocampal commissure and a rudiment of the corpus callosum body was detectable. Postnatal MR imaging, when available, confirmed prenatal forebrain commissure findings.

CONCLUSIONS: Most fetuses with apparent isolated agenesis of the corpus callosum showed at least 1 forebrain commissure at prenatal MR imaging, and approximately half of fetuses also had a second commissure: a vestigial hippocampal commissure or a hybrid made of a hippocampal commissure and a rudimentary corpus callosum body. Whether such variability is the result of different genotypes and whether it may have any impact on the long-term neurodevelopmental outcome remains to be assessed.

ABBREVIATIONS: $\mathrm{AC}=$ anterior commissure; $\mathrm{ACC}=$ agenesis of the corpus callosum; $\mathrm{CC}=$ corpus callosum; $\mathrm{GA}=$ gestational age; $\mathrm{HC}=$ hippocampal commissure; $\mathrm{HS}=$ hybrid structure

T he corpus callosum (CC) is the major white matter forebrain commissure. Agenesis of the corpus callosum (ACC) is among the most common congenital brain anomalies, often coexisting with chromosomal or genetic syndromes and other malformations of the central nervous system or extra-CNS location, ${ }^{1-4}$ which may negatively impact the neurodevelopmental outcome. $^{5-7}$ MR imaging ${ }^{8,9}$ has been introduced in prenatal assessment of suspected ACC as a complementary investigation to

Received July 6, 2015; accepted after revision August 7.

From the Radiology and Neuroradiology Department (C.C., C.P., G.C., A.R.), Children's Hospital V. Buzzi, Milan, Italy; Medical Genetics Unit (C.C.), Fondazione Istituto di Ricovero e Cura a Carattere Scientifico Ca' Granda, Ospedale Maggiore Policlinico, Milan, Italy; Fetal Medicine Unit (M.N., T.G., E.C.), S. Orsola-Malpighi Hospital, University of Bologna, Bologna, Italy; Obstetrics and Gynecology Department (M.N., G.G.), Carlo Poma Hospital, Mantova, Italy; Obstetrics Department (T.G.), Ospedale Maggiore, University of Parma, Parma, Italy; and Department of Health Sciences (G.C.), University of Milan, Milan, Italy.

Please address correspondence to Claudia Cesaretti, MD, Radiologia e Neuroradiologia Pediatrica, Ospedale dei Bambini V. Buzzi, Via Castelvetro 32, 20154 Milan, Italy; e-mail: claudia.cesaretti@gmail.com

http://dx.doi.org/10.3174/ajnr.A4570 sonography, due to its high performance in evaluating the fetal brain structures and detecting associated anomalies that may be overlooked at sonography but may impact the final outcome.

Both prenatal and postnatal MR imaging may accurately depict the brain features accompanying ACC. For example, the presence or absence of Probst bundles has been extensively addressed in the literature. ${ }^{10-12}$ On the contrary, the involvement in ACC of the other forebrain commissures, namely the anterior commissure (AC) and the hippocampal commissure (HC), has been poorly investigated and mainly as sporadic imaging reports in the postnatal setting. ${ }^{8,13}$ These postnatal reports did not provide consistent data about how the other forebrain commissures are involved in ACC. These reports are not the result of a random case screening; rather, they are a collection of clinical cases.

Consistent visualization of the other forebrain commissures seems feasible at prenatal MR imaging, albeit very poor data are available regarding the forebrain commissures in fetuses with ACC.

AJNR Am J Neuroradiol 37:521-27 Mar 2016 www.ajnr.org 
The aim of this study was to describe the types of other forebrain commissures and to assess their frequency in a large cohort of fetuses with apparent isolated ACC on prenatal MR imaging.

\section{MATERIALS AND METHODS}

The MR imaging data base of our tertiary referral center was retrospectively searched for all cases diagnosed antenatally with isolated ACC between 2004 and 2014. All cases that underwent prenatal MR imaging were referred to our hospital after expert neuroultrasonography examination.

Cases were excluded if additional brain or extra-CNS anomalies were antenatally suspected. Because of the remarkable complexity of ACC etiology and the existence of several heterogeneous syndromes under the "umbrella" definition of callosal agenesis, we chose to limit our investigation field to cases without apparent additional CNS or extra-CNS anomalies. Commonly associated findings with ACC were not considered exclusion criteria (ie, ventriculomegaly). Other features that may be frequently associated with ACC in postnatal imaging, such as hippocampal malrotation or anterior cerebral artery variability, could not be considered in our fetal imaging analysis. The relatively low mean gestational age (GA) (26 weeks) of our population prevented us from a detailed evaluation of such findings (ie, in younger fetuses, the hippocampus is still physiologically incompletely rotated). ${ }^{14}$

In the study period, MR imaging was performed on a $1.5 \mathrm{~T}$ magnet system (Achieva; Philips Healthcare, Best, the Netherlands) with body phased array coils. Standard MR imaging protocol applied to all studies was the following: a T1-weighted fast spin-echo sequence (TR/TE, 300/14 ms; turbo factor, 5; flip angle, $69^{\circ}$; number of signals acquired, 1 ; matrix, $256 \times 256$; FOV, 340 $\mathrm{mm}$; section thickness, $5.5 \mathrm{~mm}$; intersection gap, $4 \mathrm{~mm}$; number of sections, 6; acquisition time, 14 seconds) during maternal apnea; a T2-weighted single-shot fast spin-echo sequence (TR/TE, $3000 / 180 \mathrm{~ms}$; turbo factor, 85 ; flip angle, $90^{\circ}$; matrix, $256 \times 256$; FOV, $260 \mathrm{~mm}$; section thickness, 3-4 mm; intersection gap, 0.1 mm; number of sections, 11; acquisition time, 12 seconds); and a balanced fast-field echo sequence, with 2-mm-thick sections especially on the sagittal plane. All women underwent fetal MR imaging without sedation. The acquisition time of fetal scanning ranged between 20 and 30 minutes.

Despite our cases being collected during an entire decade, the single-shot fast spin-echo T2-weighted image quality has been quite constant with time, because, unfortunately, the technology of single-shot $k$-space sampling has not changed significantly since its introduction, not allowing a progression of in-plane spatial resolution.

Two pediatric neuroradiologists with $>10$ years' experience (A.R. and C.P.) reviewed the MR imaging examination of each fetus, first confirming the absence of other brain anomalies associated with ACC and then assessing the presence or absence of other forebrain commissures.

The following forebrain commissures were assessed in the 2 planes: the AC (defined as a tubularlike structure connecting the 2 hemispheres and located anterior and inferior to the thalamic mass); the HC (defined as any structure connecting the right and left fornical crura of the hippocampus and located anteriorly and at the level of the thalamic mass convexity); and the hybrid struc- ture (HS; defined as an ovoid vestigial structure resembling a CC remnant located immediately beside the $\mathrm{AC}$, anterior and wellcranial to the thalamic mass convexity). In the group with the HS, a line intersecting the mammillary body and the AC was traced on the midsagittal section to differentiate an HS from a CC genu remnant, according to the method reported by Kier and Truwit. ${ }^{15}$

The presence of an interthalamic adhesion, an interhemispheric structure that connects the thalami, was also assessed.

Because the occurrence of some degree of ventriculomegaly is frequent in isolated ACC, the hypothesis that commissural variability may correlate with different ventricular sizes was tested. In all cases, the diameter of both lateral ventricles was measured on coronal sections through the atrial level. The average of the ventricle diameters was used for statistical analysis.

If the pregnancy was not terminated, the antenatal findings were reassessed after birth by cerebral sonography or MR imaging. Postnatal MR imaging included at least sagittal T1-weighted and axial and coronal T2-weighted sections.

This retrospective study complied with clinical review study guidelines in use in our institution. A specific signed consent form for prenatal MR imaging retrospective studies was obtained from each woman undergoing prenatal MR imaging.

The Mann-Whitney $U$ test was used to compare the medians of variables that were not parametric among different groups. The $\chi^{2}$ test was used to compare qualitative data among different groups. $P<.05$ was considered statistically significant. All statistical analyses were performed by using SPSS, Version 18.0 (IBM, Armonk, New York).

\section{RESULTS}

Overall, 62 fetuses ( 32 males, 30 females; mean gestational age, 26 weeks; range, 20-35 weeks) with an antenatal diagnosis of isolated ACC at prenatal MR imaging were retrieved from our archive, obtained from 61 pregnancies. Two were twin pregnancies, one with both fetuses showing ACC, the other with only 1 fetus affected.

In all 62 fetuses, ACC was confirmed at imaging review as not being associated with other brain anomalies, with the exception of concomitant colpocephaly and ventriculomegaly, which were identified in 35 cases (56.5\%).

From the entire study group, 48 live births were registered, whereas pregnancy was electively terminated upon couple request in 14 cases.

In $32 / 62$ cases (51.6\%), the karyotype was obtained by an antenatal invasive procedure or postnatal blood sampling: Thirty showed normal chromosomic analysis findings, while in 2 cases, a trisomy 8 mosaicism was detected. Normal findings on arraycomparative genomic hybridization analysis were observed in 2 cases showing normal karyotypes.

All cases were divided into 4 groups (Fig 1 ) according to the presence or absence of other forebrain commissures. Three cases $(4.8 \%)$ did not show any forebrain commissure (group 1) (Fig 2); 23 cases $(37.1 \%$ ) presented only the AC (group 2) (Fig 3); 20 cases (32.3\%) showed an AC and HC (group 3) (Fig 4); and in the remaining 16 cases $(25.8 \%)$, the AC and an ovoid HS were detected (group 4) (Fig 5). In all 16 cases, the mentioned ovoid HS appeared to be located well posterior to the line 
intersecting the mammillary body and the $\mathrm{AC}$ on the midsagittal section, thus excluding the possibility of a CC genu remnant, according to the model of Kier and Truwit. ${ }^{15}$

Interthalamic adhesion was not detected in 14/62 fetuses (22\%), 9 of group 2, 2 of group 3, and 3 of group 4, without a statistically significant difference in the prevalence among the 4 groups $(P=.09)$.

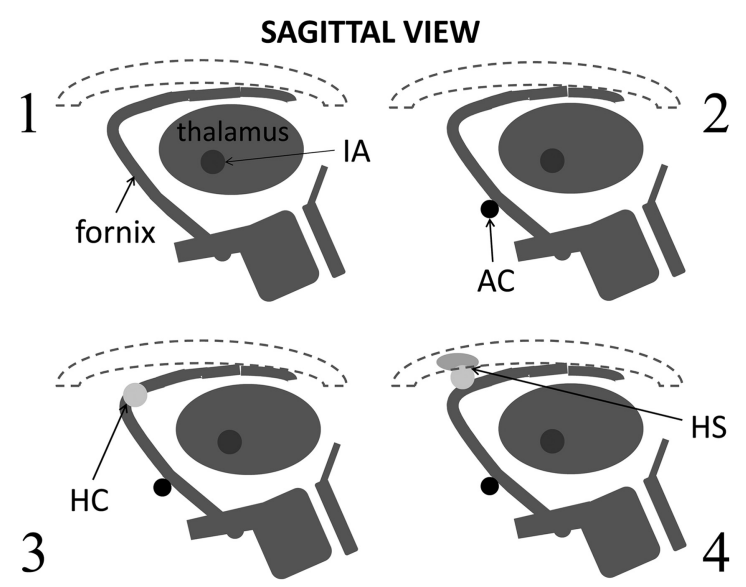

FIG 1. A scheme showing 4 sagittal view examples of the corpus callosum putative region, reporting the 4 groups on the basis of forebrain commissures. IA indicates interthalamic adhesion.
Because in our cohort, the major morphologic difference among the 4 groups seemed to be related to the presence or absence of a second commissural structure in addition to the AC itself, we compared group 2 with groups 3 and 4 pooled together in terms of atrial ventricle diameter. The median GA at MR imaging of group 2 (27 weeks; range, 20-34 weeks) and groups 3 and 4 pooled together (23 weeks; range, $20-35$ weeks) did not show a statistically significant difference $(P=.29)$; the same result $(P=.12)$ was obtained comparing the atrial lateral ventricle diameters of group 2 (median, $14 \mathrm{~mm}$; range 5-19 $\mathrm{mm}$ ) with those of groups 3 and 4 pooled together (median, 11 $\mathrm{mm}$; range, $8-26 \mathrm{~mm}$ ).

In all neonates, cranial sonography was available and did not show additional brain malformations. A postnatal MR imaging study was available in 22 cases, and in all, the prenatal features related to the commissural structures were confirmed. In 3 cases of group 4, diffusion tensor-based fiber-tracking was obtained and it confirmed that the HS was composed of fibers connecting both the fornical crura and the hemispheric parenchyma (Fig 5).

\section{DISCUSSION}

Our study shows that prenatal MR imaging can identify different classes of isolated ACC on the basis of the presence or absence of other forebrain commissural structures. Because the approach of
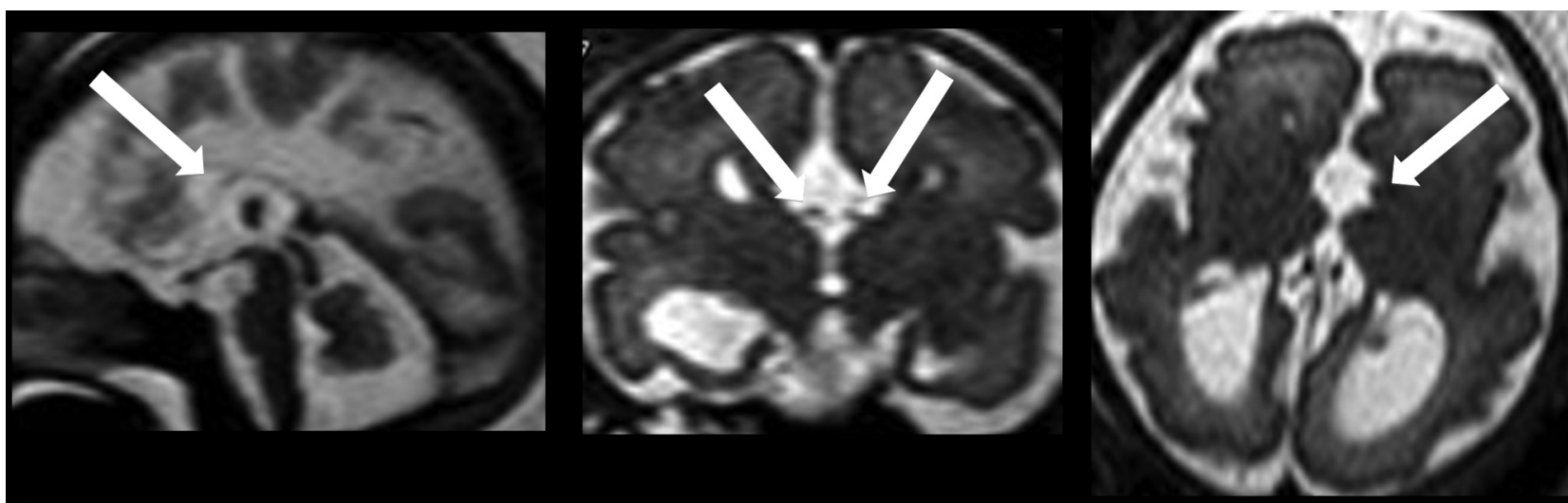

A

B

C

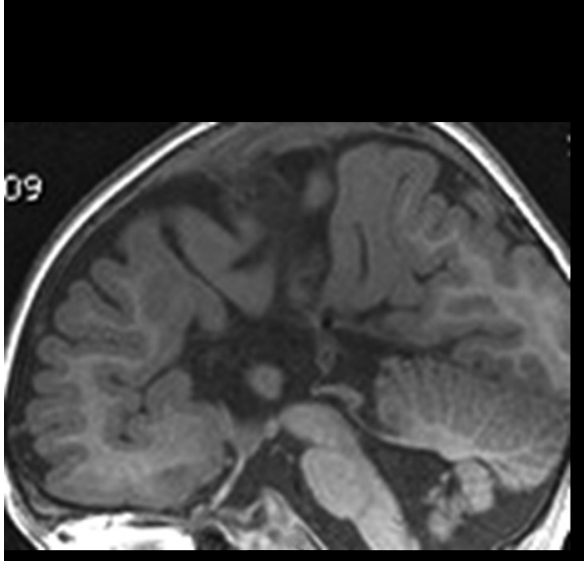

D
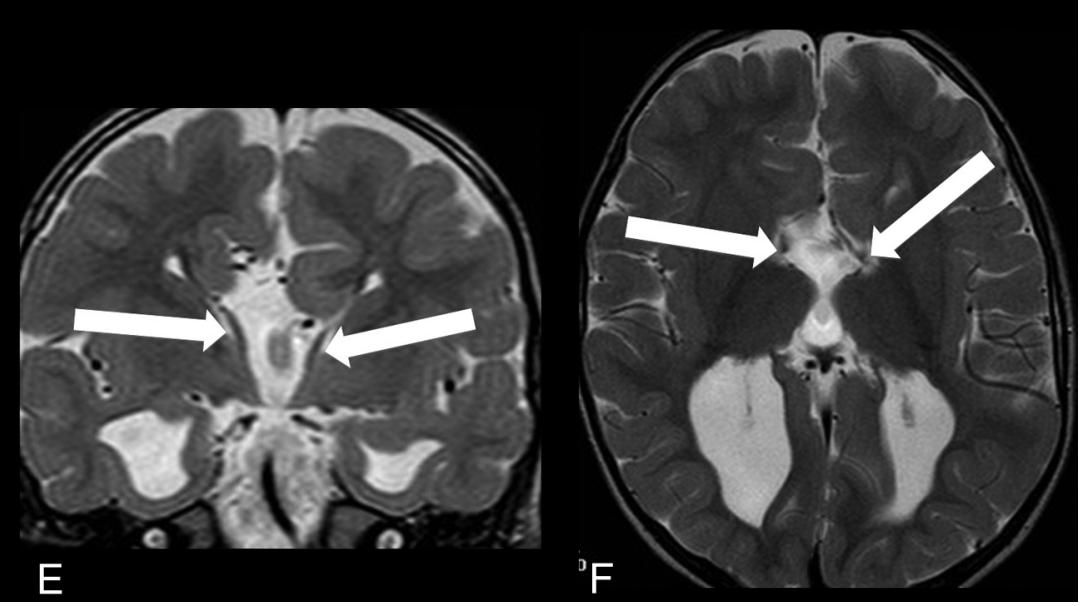

FIG 2. Group 1 fetuses. A-C, Sagittal, coronal, and axial single-shot fast spin-echo 3-mm-thick T2-weighted sections from a 32-week-GA study with no visible AC or HC. White arrows show the fornical crura. $D-F$, Sagittal T1 and coronal and axial T2-weighted sections from a postnatal study confirm the prenatal findings. White arrows show the fornical crura with no HC connecting them. 


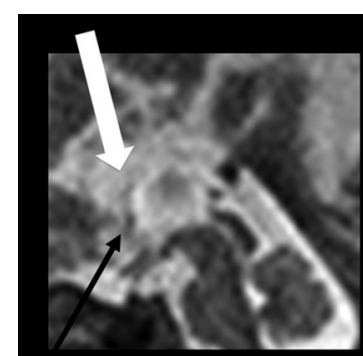

A

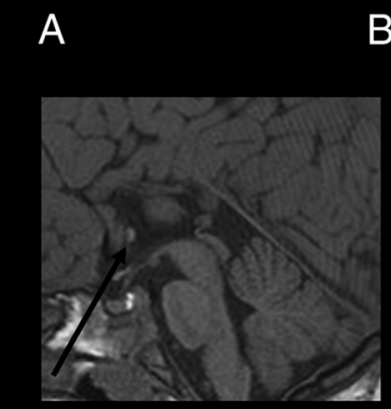

F

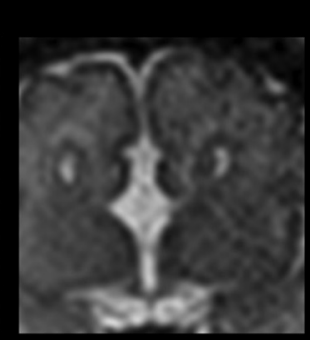

B

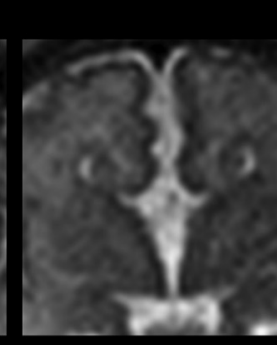

C

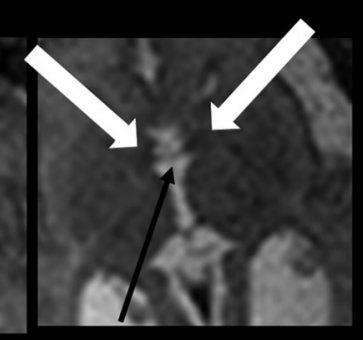

D

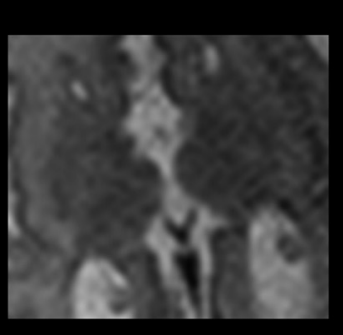

E

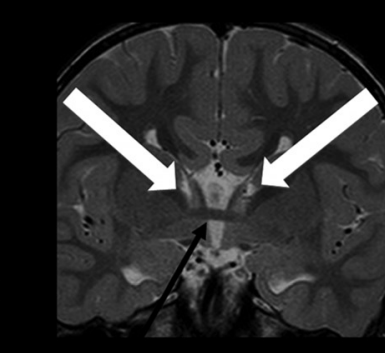

G

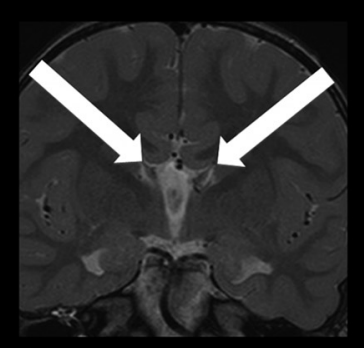

$\mathrm{H}$

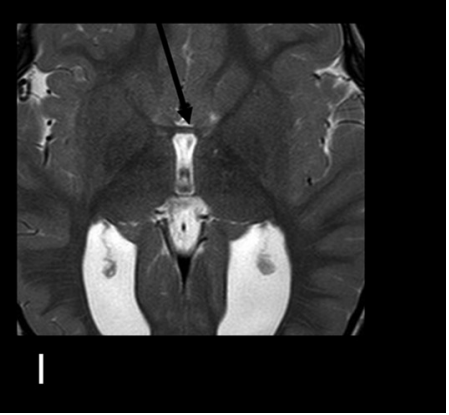

FIG 3. Group 2 fetuses. A-E, Sagittal, coronal, and axial single-shot fast spin-echo 3-mm-thick T2-weighted sections from a 30-week-GA study with a visible AC (black arrows) but no HC. White arrows show the fornical crura. F-l, Sagittal T1 and coronal and axial T2-weighted sections from a postnatal study confirm prenatal findings: the presence of only the AC (black arrows). White arrows show the fornical crura with no HC connecting them.

this study was based on randomized recruitment of cases, our data are likely to provide information about the real frequencies of ACC subgroups according to the types of other forebrain commissures.

Commissures are bundles of white matter that cross the midline and connect symmetric structures on the 2 cerebral hemispheres. The CC is the major white matter commissure between the 2 cerebral hemispheres, extending from the frontal lobe anteriorly to above the collicular plate posteriorly. Apart from the CC, there are 2 main interhemispheric forebrain commissures: the $\mathrm{AC}$ and the $\mathrm{HC}$, the latter being, in healthy cases, a lamina lying underneath the callosal splenium. ${ }^{13}$

According to the extensive review of Raybaud, ${ }^{13}$ there is an intimate relation between the early developing body of the CC and the vestigial $\mathrm{HC}$ in the fetal brain, starting from 11-13 weeks of GA. The HC, which is initially located in the anterior third ventricle area, is the archipallian commissure between hemispheres. It assumes tubular (on coronal view) and ovoid (on sagittal view) shapes. Early HC supports the development of the very early body of the CC; the latter is located immediately above the vestigial $\mathrm{HC}$, with interposition of a glial sling. The HC then progressively migrates and elongates toward its final subsplenial position; the body of the CC courses parallel to the HC in its development.

In our series, we identified 4 groups of fetuses with ACC based on the presence or absence of other forebrain commissures: group 1: no forebrain commissure; group 2: AC only; group 3: AC and vestigial $\mathrm{HC}$; and group 4: AC and a HS, with the HS composed of a vestigial $\mathrm{HC}$ and an early rudiment of the CC body.

Only 3 of our cases presented without any forebrain commissure (group 1), supporting the hypothesis that this is actually the less common condition with respect to other ACC types.
Among the remaining 3 groups with an AC, a further division was proposed on the basis of the absence (group 2) or presence (groups 3 and 4) of some transverse structures connecting the fornical crura (HC or HS). After matching prenatal and postnatal images, we speculated that the tubular structure joining the hippocampal fornical crura might be a form of vestigial dysmorphic $\mathrm{HC}$ that did not complete development toward its final normal laminar shape and its normal location underneath the splenium and posterior third of CC. In this regard, we did not encounter, in our cohort, a single ACC case with a normal laminar-shaped HC. On the other side, the ovoid structure described well above the thalamic mass convexity could represent an HS composed of a very initial underdeveloped CC component (rudiment) merged, in some cases, with a vestigial $\mathrm{HC}$ component. In particular, by tracing a line according to the method reported by Kier and Truwit, ${ }^{15}$ we demonstrated that this rudimentary structure resides posterior to such a line in all cases of group 4 (Fig 5). This location may suggest that such an abnormal structure is compatible with a very early component of the developing body of the CC, rather than with a genu remnant.

The ovoid structure reported in group 4 has totally different characteristics with respect to the diagonal aberrant oblique tracts reported recently in some cases of CC dysgenesis ${ }^{11,12}$; in our cases, the vestigial structure is clearly orthogonal to the cerebral hemispheres and does not resemble an X-shaped bundle, as confirmed by postnatal fiber-tracking studies (Fig 5).

The interesting work of Kasprian et al, ${ }^{11}$ which highlighted the possibility of Probst bundles depicted through fiber-tracking technique during intrauterine life, does not seem to be applicable to study the AC and the vestigial HC because the currently limited diffusion tensor imaging spatial resolution (approximately 2 $\mathrm{mm}^{2}$ ) would not intercept these commissures. Even though our 


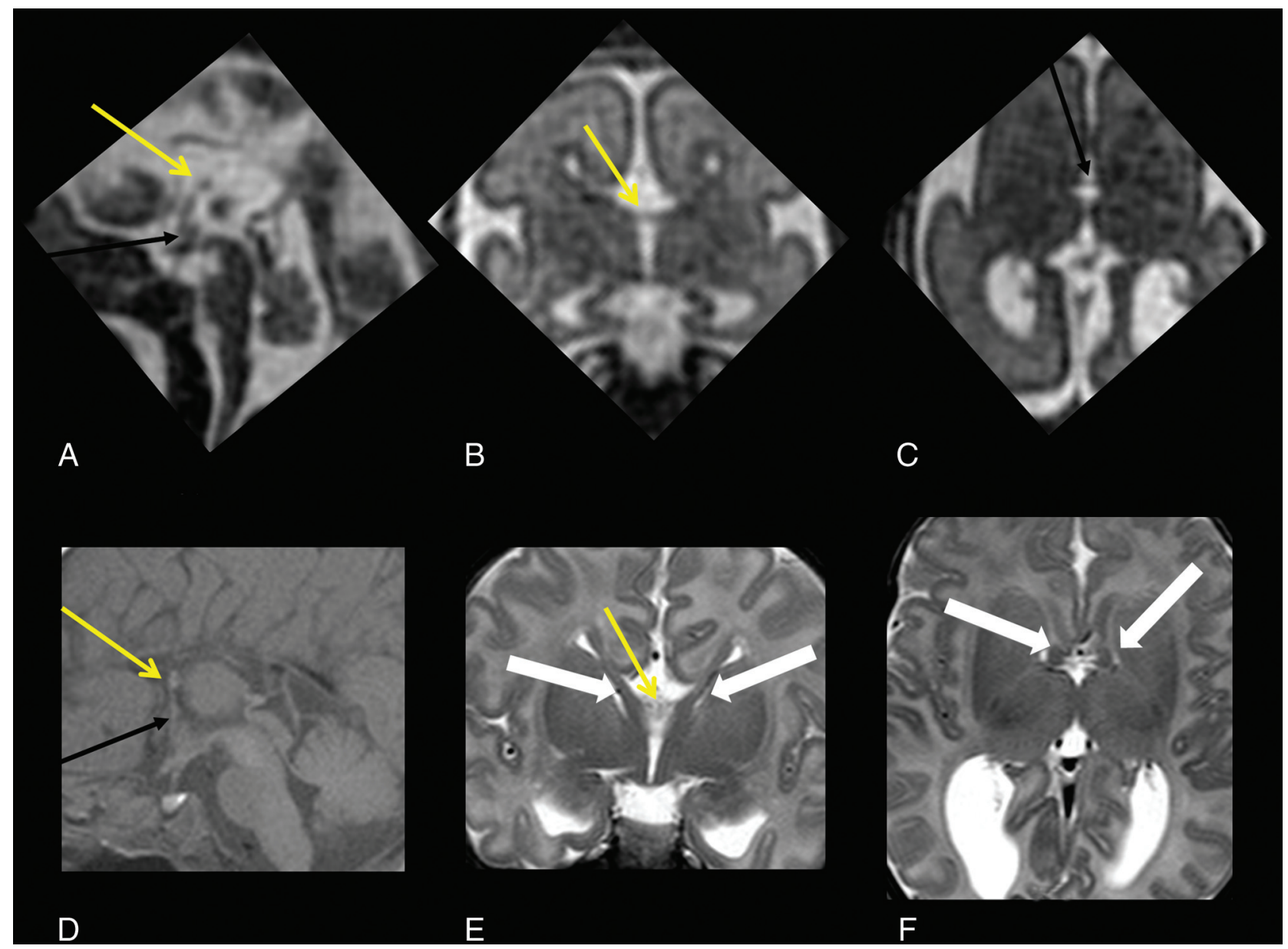

FIG 4. Group 3 fetuses. A-C, Sagittal, coronal, and axial single-shot fast spin-echo 3-mm-thick T2-weighted sections from a 27-week-GA study with a visible vestigial $\mathrm{HC}$ (yellow arrows). Black arrows show the AC. D-F, Sagittal T1 and coronal and axial T2-weighted sections from the postnatal study confirm the prenatal findings: the presence of the AC (black arrow) and a vestigial HC (yellow arrows), connecting the fornical crura (white arrows).

imaging protocol included sections not thinner than $2 \mathrm{~mm}$, the identification of commissural structures was obtained with confidence by the 2 readers by comparing multiple sets of coronal, axial, and sagittal images, because at least 3 series of sections had been acquired for each plane redundantly. In particular, the AC, which is the thinnest of the commissures, when not clearly appreciable in axial or coronal planes, could be usually visualized on the sagittal plane, taking advantage of the higher in-plane spatial resolution (about $1 \mathrm{~mm}^{2}$ ).

Due to the frequent association of isolated ACC with ventriculomegaly, we further investigated whether this finding could have any relationship with the presence of $\mathrm{HC}$ or HS. Our results showed no statistical difference of the atrial width between group 2 and groups 3 and 4 pooled together, thus suggesting that the presence of HC or HS has no impact on the degree of concomitant ventriculomegaly.

Regarding the prevalence of interthalamic adhesion in our cases, we found that our data are very similar to those reported by previous imaging studies, which demonstrated its absence in approximately $22 \%$ of healthy subjects. ${ }^{16}$ However, we did not find any correlation with commissural variability.

The strength of this study lies in its original content, to our knowledge, being the first time that additional forebrain commis- sures have been systematically assessed at prenatal MR imaging in a large cohort of fetuses with isolated ACC and enrolled as the result of a prenatal screening.

Very recently, some neurosonographers have demonstrated, in a smaller cohort of fetuses with ACC, that HC identification was feasible by state-of-the-art 3D sonography; however, different from our study, a complete assessment of commissural variability, also including the AC, did not seem achievable. ${ }^{17}$

The major limitation of this study is the lack of postnatal MR imaging in all cases because some neonates were submitted only to cranial sonography and some pregnancies were terminated, with brain deterioration not allowing an accurate postmortem analysis.

The lack of postnatal MR imaging control in all our cases does not allow considering our report as a definite indicator of the diagnostic accuracy of prenatal MR imaging concerning the status of forebrain commissures.

The clinical and prognostic meaning of forebrain commissures variability in isolated ACC needs to be tested by long-term clinical follow-up studies, which may also include global white matter pathway rearrangement evaluation via high-resolution DTI tractography. Very recently, Meoded et $\mathrm{al}^{18}$ performed a structural connectivity study in children with isolated ACC and 


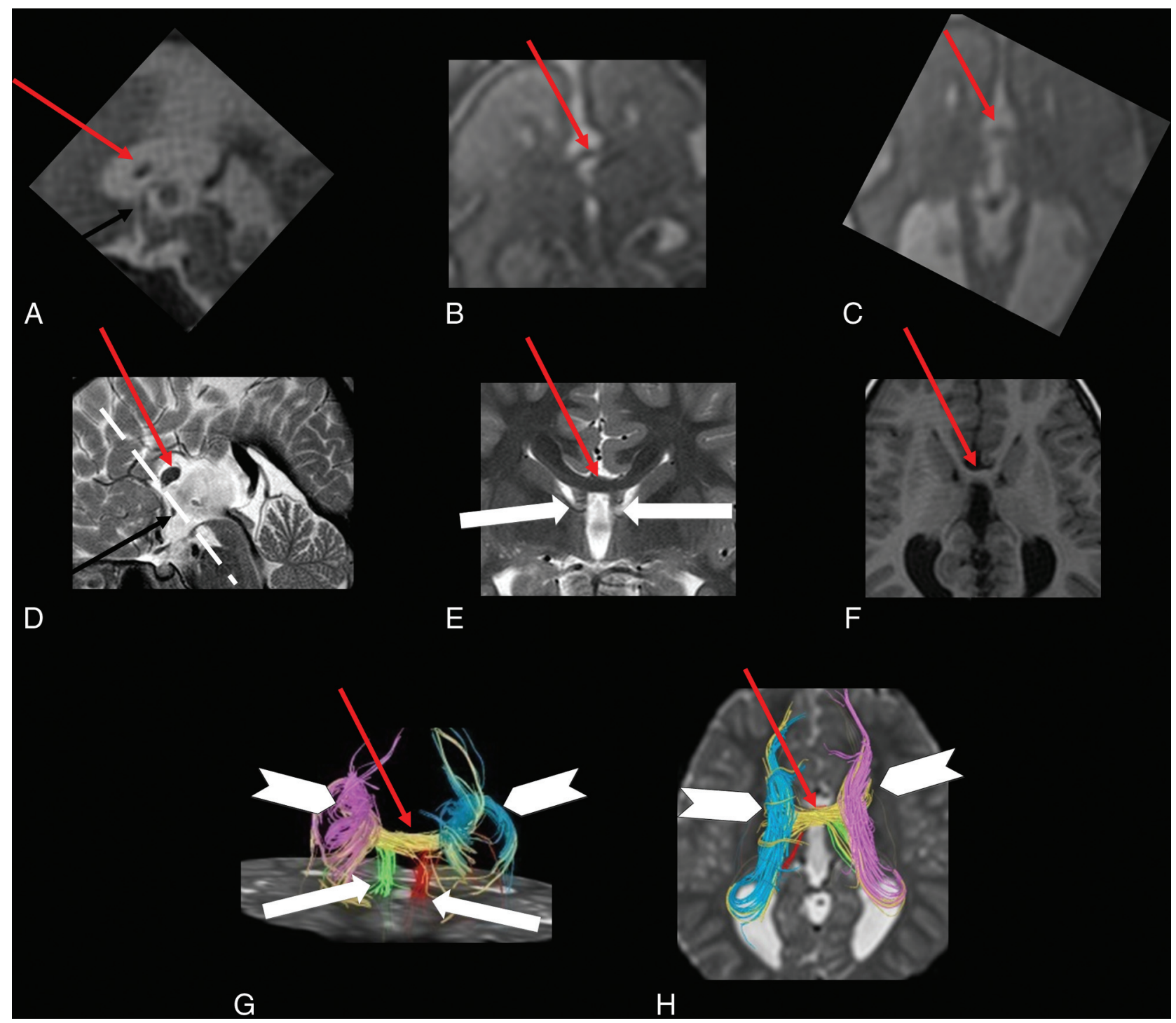

FIG 5. Group 4 fetuses. A-C, Sagittal, coronal, and axial single-shot fast spin-echo 3-mm-thick T2-weighted sections from a 25 -week-GA study with a visible AC (black arrow) and a putative HS composed of a vestigial $\mathrm{HC}$ and an early rudiment of the CC body (red arrows). This putative HS seems to be located slightly more cranially compared with the simple vestigial HC shown in Fig 3. D-F, Sagittal and coronal T2 and axial T1-weighted sections from a postnatal study confirm prenatal findings: the presence of the AC (black arrow) and a putative HS (red arrows). White arrows show the fornical crura; the dotted line in $D$ intersects the mammillary body and the AC, according to the method reported by Kier and Truwit. ${ }^{15} \mathrm{G}$ and $\mathrm{H}$, Fiber-tracking images from the same study show fibers forming the putative HS (red arrows indicate yellow fibers apparently connecting the cerebral hemispheres and also the fornical crura). Arrowheads indicate Probst bundles (blue and violet fibers), and white arrows show the fornical crura (green and red fibers).

identified hubs in the bilateral insular regions that are connected to each other by the AC, suggesting how brain plasticity may induce alternative pathways for interhemispheric connectivity. However, the clinical impact of these observations is still unknown.

\section{CONCLUSIONS}

Our data demonstrate that prenatal MR imaging may identify different groups of isolated ACC according to the presence or absence of other forebrain commissures, such as the AC, HC, and HS. Most fetuses with apparent isolated ACC showed at least the $\mathrm{AC}$ at prenatal MR imaging, and approximately half of these fetuses also showed a second commissure: a vestigial HC or an HS composed of an HC and a rudimental CC body.

Further studies are needed to ascertain whether such variabil- ity may be associated with different genetic characteristics and if it may have any impact on the long-term neurodevelopmental outcome.

\section{REFERENCES}

1. Grogono JL. Children with agenesis of the corpus callosum. Dev Med Child Neurol 1968;10:613-16 Medline

2. Jeret JS, Serur D, Wisniewski K, et al. Frequency of agenesis of the corpus callosum in the developmentally disabled population as determined by computerized tomography. Pediatr Neurosci 19851986;12:101-03 Medline

3. Glass HC, Shaw GM, Ma C, et al. Agenesis of the corpus callosum in California 1983-2003: a population-based study. Am J Med Genet $A$ 2008;146A:2495-500 CrossRef Medline

4. Santo S, D'Antonio F, Homfray T, et al. Counseling in fetal 
medicine: agenesis of the corpus callosum. Ultrasound Obstet Gynecol 2012;40:513-21 CrossRef Medline

5. Sotiriadis A, Makrydimas G. Neurodevelopment after prenatal diagnosis of isolated agenesis of the corpus callosum: an integrative review. Am J Obstet Gynecol 2012;206:337.e1-5 CrossRef Medline

6. Moutard ML, Kieffer V, Feingold J, et al. Isolated corpus callosum agenesis: a ten-year follow-up after prenatal diagnosis (how are the children without corpus callosum at 10 years of age?). Prenat Diagn 2012;32:277-83 CrossRef Medline

7. Noguchi R, Abe K, Hamada H, et al. Outcomes of patients with prenatally diagnosed agenesis of the corpus callosum in conjunction with ventriculomegaly. Arch Gynecol Obstet 2014;290:237-42 CrossRef Medline

8. Küker W, Mayrhofer H, Mader I, et al. Malformations of the midline commissures: MRI findings in different forms of callosal dysgenesis. Eur Radiol 2003;13:598-604 Medline

9. Manfredi R, Tognolini A, Bruno C, et al. Agenesis of the corpus callosum in fetuses with mild ventriculomegaly: role of MR imaging. Radiol Med 2010;115:301-12 CrossRef Medline

10. Tovar-Moll F, Moll J, de Oliveira-Souza R, et al. Neuroplasticity in human callosal dysgenesis: a diffusion tensor imaging study. Cereb Cortex 2007;17:531-41 Medline

11. Kasprian G, Brugger PC, Schopf V, et al. Assessing prenatal white matter connectivity in commissural agenesis. Brain 2013;136: 168-79. CrossRef Medline
12. Edwards TJ, Sherr EH, Barkovich AJ, et al. Clinical, genetic and imaging findings identify new causes for corpus callosum development syndromes. Brain 2014;137(pt 6):1579-613 CrossRef Medline

13. Raybaud C. The corpus callosum, the other great forebrain commissures, and the septum pellucidum: anatomy, development, and malformation. Neuroradiology 2010;52:447-77 CrossRef Medline

14. Righini A, Zirpoli S, Parazzini C, et al. Hippocampal infolding angle changes during brain development assessed by prenatal MR imaging. AJNR Am J Neuroradiol 2006;27:2093-97 Medline

15. Kier EL, Truwit CL. The normal and abnormal genu of the corpus callosum: an evolutionary, embryologic, anatomic, and MR analysis. AJNR Am J Neuroradiol 1996;17:1631-41 Medline

16. Nopoulos PC, Rideout D, Crespo-Facorro B, et al. Sex differences in the absence of massa intermedia in patients with schizophrenia versus healthy controls. Schizophr Res 2001;48:177-85 CrossRef Medline

17. Contro E, Nanni M, Bellussi F, et al. The hippocampal commissure: a new finding at prenatal 3D ultrasound in fetuses with isolated complete agenesis of the corpus callosum. Prenat Diagn 2015;35: 919-22 CrossRef Medline

18. Meoded A, Katipally R, Bosemani T, et al. Structural connectivity analysis reveals abnormal brain connections in agenesis of the corpus callosum in children. Eur Radiol 2015;25:1471-78 CrossRef Medline 